\title{
Analysis of Natural Convection Flow in a Trapezoidal Cavity Containing a Rectangular Heated Body in Presence of External Oriented Magnetic Field
}

\author{
A. Akter ${ }^{*}$, S. Parvin \\ Department of Mathematics, Bangladesh University of Engineering and Technology, Dhaka-1000, \\ Bangladesh
}

Received 31 August 2017, accepted in final revised form 21 December 2017

\begin{abstract}
The laminar natural convection flow and heat transfer inside a trapezoidal cavity filled with air and containing a rectangular block is investigated numerically. The left and right walls of the cavity are cold, the top and bottom walls are adiabatic and the rectangular body is heated uniformly. Finite Element Method of Galerkin's weighted residual scheme is used to solve the transport equations with appropriate boundary conditions. The main objective of this study is to explore the influence of pertinent parameters such as Rayleigh number $(R a)$, Hartmann number $(\mathrm{Ha})$ and orientation of the magnetic field $(\phi)$ on the flow and heat transfer performance of the fluid while the Prandtl number $(P r)$ is considered fixed. Results indicate that the heat transfer rate is significantly affected by increasing the mentioned parameters.
\end{abstract}

Keywords: Natural convection; Trapezoidal cavity; Magnetic field.

(C) 2018 JSR Publications. ISSN: 2070-0237 (Print); 2070-0245 (Online). All rights reserved. doi: http://dx.doi.org/10.3329/jsr.v10i1.33848 J. Sci. Res. 10 (1), 11-23 (2018)

\section{Introduction}

Analysis of convection such as natural convection in enclosed cavities usually is used in the engineering fields. Most of the cavities commonly used in industries are circular, square, rectangular, trapezoidal and triangular etc. Although many works have been conducted using rectangular cavities, very limited works have been done using trapezoidal cavity. Hossain et al. [1] analyzed numerical analysis on MHD natural convection within trapezoidal cavity having circular block. They have seen that for different inclination angles conduction dominant region changes for different $\operatorname{Pr}$ when $R a$ increases. Esfe et al. [2] studied natural convection in a trapezoidal enclosure filled with carbon nanotube-EGwater nanofluid. They investigated that at low Rayleigh number, the average Nusselt number decreases with increasing the inclination angle at all solid volume fractions again for increasing Rayleigh number, the average Nusselt number increases and then decreases

\footnotetext{
Corresponding author: afrozamath@math.buet.ac.bd
} 
with inclination angle. Effect of magnetic field on natural convection flow in a prism shaped cavity filled with nanofluid was investigated by S. Parvin and A. Akter [3]. They also studied numerical analysis of heat generation effect on natural convection flow in a trapezoidal cavity containing a rectangular heated body [4]. Selimefendigil et al. [5] investigated entropy generation due to natural convection in entrapped trapezoidal cavities filled with nanofluid under the influence of magnetic field where the upper (lower) cavity is filled with $\mathrm{CuO}$-water $\left(\mathrm{Al}_{2} \mathrm{O}_{3}\right.$-water $)$ nanofluid and the top and bottom horizontal walls of the trapezoidal cavities are maintained at constant hot temperature while other inclined walls of the enclosures are at constant cold temperature and also different combinations of Hartmann numbers are imposed on the upper and lower trapezoidal cavities. It is found that the heat transfer enhancement rates with nanofluids which are in the range of $10 \%$ and $12 \%$ are not affected by the presence of the magnetic field. Buoyancy induced fluid flow and heat transfer in inclined trapezoidal cavity was analyzed by Lee [6]. He analyzes the behavior of the flow and heat transfer characteristics at different Rayleigh and Prandtl numbers in his numerical study. Hasanuzzaman et al. [7] studied a computational numerical work to see the effects of magnetic field on natural convection for a trapezoidal cavity. Nasrin and Parvin [8] conducted a numerical research in order to investigate the transport mechanism of natural convection in a trapezoidal cavity filled with $\mathrm{Cu}-$ water nanofluid. They found that both aspect ratio and Prandtl number affect the fluid flow and heat transfer in the cavity. In addition they developed a correlation for the average Nusselt number as a function of the Prandtl number as well as the cavity aspect ratio. Ellahi [9] studied magneto-hydrodynamic (MHD) flow of non-Newtonian nanofluid in a pipe and observed that the MHD parameter decreases the fluid motion and the velocity profile is larger than that of the temperature profile even in the presence of variable viscosities. Free convection heat transfer in a concentric annulus between a cold square and heated elliptic cylinders in the presence of a magnetic field was investigated by Sheikholeslami et al. [10]. They found that enhancement in heat transfer increases as the Hartmann number increases but it decreases with increase of Rayleigh number. The flow within an enclosure consisting of a prism shaped body at different temperatures is an important circumstance encountered quite frequently in practice.

There are plentiful studies in the literature regarding natural convection in enclosures. The phenomenon of heat and fluid flow for a configuration of isothermal vertical walls, controlled at different temperatures and with adiabatic horizontal walls are well explained [11]. Parvin and Nasrin [12] studied the effects of Reynolds and Prandtl number on mixed convection in an octagonal channel with a heat-generating hollow cylinder. Most of the previous studies on natural convection in enclosures are related to either side heating or bottom heating [10-16]. Chowdhury et al. [17] has analyzed heat generation effect on natural convection flow in closed cavities. They observed that the rate of heat transfer decreases with the increasing of heat generation. Sheremet et al. [18] investigated the numerical analysis of entropy generation in natural convection of nanofluid inside a square cavity having hot solid block. They develop the ability to control the flow via an external magnetic field to have an optimal flow by decreasing the entropy generation. 
Use of an external magnetic field is of considerable importance in many industrial applications, particularly as a control mechanism in material manufacturing. Jery et al. [19] studied effect of an external oriented magnetic field on entropy generation in natural convection. They found that the magnetic field tends to decrease the convection currents, the heat transfer and entropy generation inside the enclosure. Hayat et al. [20] analyzed effect of inclined magnetic field in flow of third grade fluid with variable thermal conductivity. Here they examine effects of inclined magnetic field and heat transfer in the flow of a third-grade fluid by an exponentially stretching surface. Sandeep and Sugunamma [21] analyze the effects of inclined magnetic field and radiation on free convective flow of dissipative fluid past a vertical plate through porous medium in presence of heat source. Baskaya et al. [22] studied the investigation of oriented magnetic field effects on entropy generation in an inclined channel filled with ferrofluids. This study investigates ferrofluid flow characteristics in an inclined channel under inclined magnetic field and constant pressure gradient. Mehrez et al. [23] studies the MHD effects on heat transfer and entropy generation of nanofluid flow in an open cavity in the presence of an inclined magnetic field.

From the above literature review it is seen that trapezoidal cavity having a heated rectangular body in presence of the magnetic field have not been studied yet. The objective of this work is to study natural convection in a trapezoidal shaped enclosure having a rectangular heated block inside with oriented magnetic field effect. The study focuses specifically on the effects of the convective parameter on the streamlines, isothermal distribution and average Nusselt number.

\section{Model and Mathematical Formulation}

Two dimensional trapezoidal cavity of height $H$ and base $L$ whose lower left corner point is located at $(0,0)$ is considered with the boundary conditions. A heated rectangular body of base $l_{x}$ and height $h_{y}$ is inserted in the cavity whose lower left corner is located at the point $(0.5,0.2)$ and is maintained at temperature $T_{h}$. The left and right wall is maintained at cold temperature $T_{c}$ where $T_{h}>T_{c}$. Top and bottom walls are adiabatic. The cavity is permeated by the uniform oriented magnetic field $\boldsymbol{B}=B_{x} \boldsymbol{e}_{x}+B_{y} \boldsymbol{e}_{y}$ (where $B_{x}$ and $B_{y}$ are space independent) of constant magnitude $B_{0}=\sqrt{B_{x}^{2}+B_{y}^{2}}$ and $\boldsymbol{e}_{x}$ and $\boldsymbol{e}_{\boldsymbol{y}}$ are unit vectors in Cartesian coordinate system. The orientation of the magnetic field forms an angle $\phi$ with horizontal axis, such that, $\phi=\frac{B_{y}}{B_{x}}$.

In the present study, we assume steady, two-dimensional, laminar flow of a viscous incompressible fluid having constant properties. Under Boussinesq approximation the dimensionless equations of mass, momentum and energy are as follows:

$$
\begin{aligned}
& \frac{\partial U}{\partial X}+\frac{\partial V}{\partial Y}=0 \\
& U \frac{\partial U}{\partial X}+V \frac{\partial U}{\partial Y}=-\frac{\partial P}{\partial X}+\operatorname{Pr}\left(\frac{\partial^{2} U}{\partial X^{2}}+\frac{\partial^{2} U}{\partial Y^{2}}\right)+H a^{2}\left(V \sin \phi \cos \phi-U \sin ^{2} \phi\right)
\end{aligned}
$$


$U \frac{\partial V}{\partial X}+V \frac{\partial V}{\partial Y}=-\frac{\partial P}{\partial X}+\operatorname{Pr}\left(\frac{\partial^{2} V}{\partial X^{2}}+\frac{\partial^{2} V}{\partial Y^{2}}\right)+\frac{R a}{P r} \theta+H a^{2}\left(U \sin \phi \cos \phi-V \cos ^{2} \phi\right)$

$U \frac{\partial \theta}{\partial X}+V \frac{\partial \theta}{\partial Y}=\frac{\partial^{2} \theta}{\partial X^{2}}+\frac{\partial^{2} \theta}{\partial Y^{2}}$

where,

$X=\frac{x}{L}, Y=\frac{y}{L}, U=\frac{u L}{\alpha}, V=\frac{v L}{\alpha}, \theta=\frac{T-T_{C}}{T_{h}-T_{C}}$

$P=\frac{p L^{2}}{\rho \alpha^{2}},=\frac{v}{\alpha}, R a=\frac{\mathrm{g} \beta\left(T_{h}-T_{c}\right) L^{3} P r}{v^{2}}$ and $H a^{2}=\frac{\sigma B_{0}^{2} L^{2}}{\mu}$

In above equation $X$ and $Y$ are the dimensionless distances along $x$ - and $y$-coordinate respectively, $L$ is the base of the cavity, $U$ and $V$ are the corresponding velocity components along the coordinate axes, $P$ denotes the dimensionless pressure, $\theta$ is the dimensionless temperature whereas $P r$ and $R a$ denote Prandtl and Rayleigh numbers, respectively. The boundary conditions can be summarized by the following equations:

All boundaries are rigid and no-slip: $\mathrm{U}=\mathrm{V}=0$

On the left and right inclined surface: $\theta=0$

On the top and bottom surface: $\frac{\partial \theta}{\partial n}=0$

At the rectangular body: $\mathrm{U}=\mathrm{V}=0 ; \theta=1$.

The relationships between stream function $\psi$ and velocity components $U, V$ for twodimensional flows are

$U=\frac{\partial \psi}{\partial Y}$ and $U=\frac{\partial \psi}{\partial X} \quad$ which give a single equation $\frac{\partial^{2} \psi}{\partial X^{2}}+\frac{\partial^{2} \psi}{\partial Y^{2}}=\frac{\partial U}{\partial Y}-\frac{\partial V}{\partial X}$. The no-slip condition is valid at all boundaries as there is no cross-flow. Hence $\Psi=0$ is used for boundaries.

Mean Nusselt number is calculated using, $N u=-\int_{0}^{S} \frac{\partial \theta}{\partial n} d S$

Where $\frac{\partial \theta}{\partial n}=\sqrt{\left(\frac{\partial \theta}{\partial X}\right)^{2}+}\left(\frac{\partial \theta}{\partial Y}\right)^{2}$ and $S$ is the boundary of the heated body. 


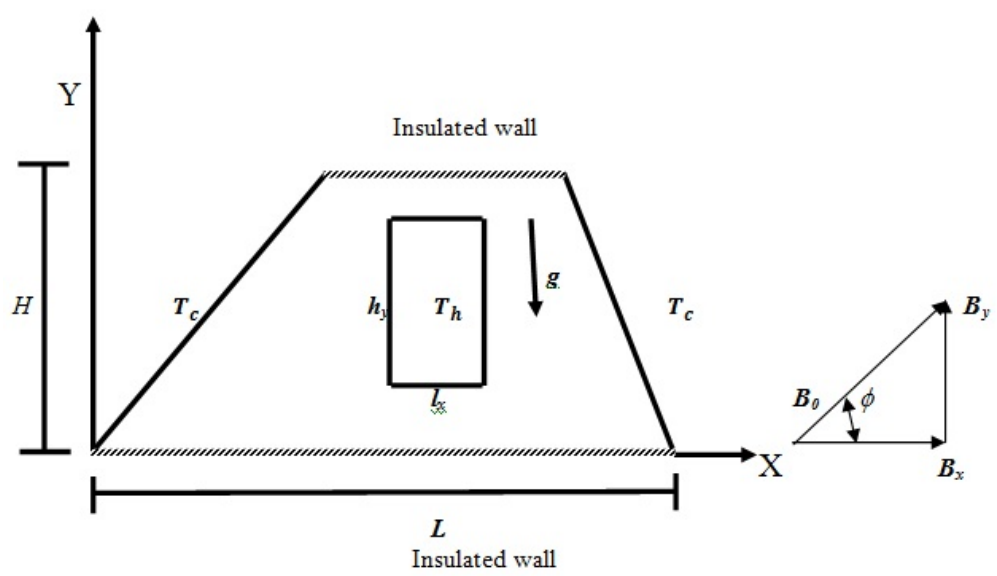

Fig. 1. Schematic diagram for the physical system.

\section{Solution Technique}

Galerkin weighted residual method of finite element is used in the study as the solution technique. In this method, the solution domain is divided into finite number of elements, which are formed of non-uniform triangular elements. Then by using Galerkin weighted residual method the governing non-linear partial differential equations (i.e. mass, momentum and energy equations) are transferred into a system of integral equations. The integration involved in each term of these equations is performed by using Gauss's quadrature method. Newton's method is used to modify the non-linear algebraic equations into linear equations with the help of the boundary conditions. Then finally we use Triangular Factorization method to solve these linear equations.

\section{Grid Independent T}

To guarantee a grid-independent solution for $R a=10^{5}, \operatorname{Pr}=0.7, \mathrm{Ha}=50$ and $\phi=0$ in a trapezoidal cavity an extensive mesh testing procedure is conducted. The following number of elements within the resolution field: 1526, 3580, 10470, 28127 and 36863 are examined. The numerical average Nusselt $(\mathrm{Nu})$ number for the above resolution field is obtained to understand the grid fineness as shown in Fig. 2. The average Nusselt numbers for 28127 elements shows a little difference with the next result. Hence, considering the non-uniform grid system of 28127 elements is preferred for the computation. 


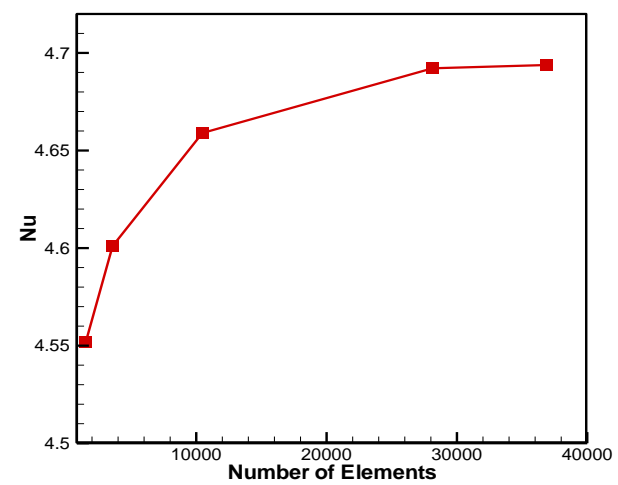

Fig. 2. Grid independent test.

\section{Model Validation}

The solution procedure for the fluid flow with the effect of circular obstacle inside a square cavity has been investigated by Parvin and Nasrin [24] for $\operatorname{Ra}=10^{5}, \mathrm{Pr}=0.7$ and $H a=50$. A test has been made to compare the results obtained by the current model with earlier study [24]. Fig. 3 represents the result of streamlines and isotherms for natural convection in a square cavity with a circular heated block. The top row presents the result of Parvin and Nasrin [24] and bottom row shows the result for current study. The comparison shows that the present's results agree with the numerical solution of Parvin and Nasrin [24].
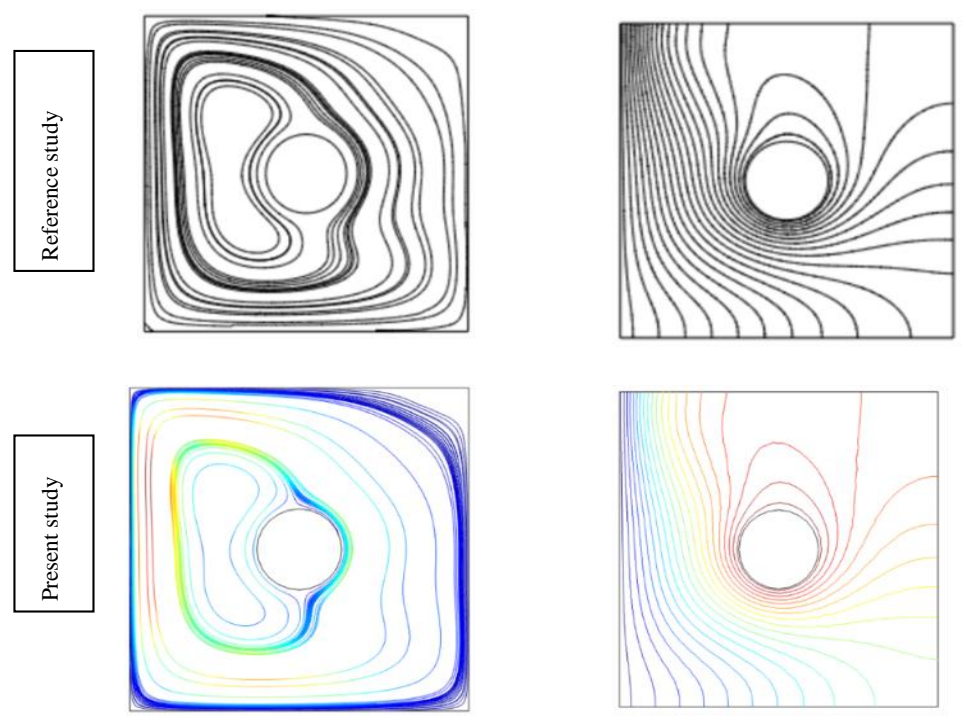

Fig. 3. A comparison for streamlines (on the left) and isotherms (on the right) between Parvin and Nasrin [24] (top row) and present study (bottom row) for $\mathrm{Ra}=10^{5}, \mathrm{Pr}=0.7$ and $\mathrm{Ha}=50$. 


\section{Results and Discussion}

The numerical computation has been carried out through the finite element method to analyze natural convection inside a trapezoidal cavity containing a rectangular heated body. Effects of the parameters such as Rayleigh number $(\mathrm{Ra})$, Hartmann number $(\mathrm{Ha})$ and Orientation of magnetic field $(\phi)$ on heat transfer and fluid flow inside the cavity has been studied. The ranges of $\mathrm{Ra}, \mathrm{Ha}$, and $\phi$ for this investigation vary from $\left(10^{3}\right.$ to $\left.10^{6}\right),(0$ to 70$)$ and $\left(0^{\circ}\right.$ to $\left.90^{\circ}\right)$ respectively while the Prandtl number is kept fixed at $\mathrm{Pr}=0.7$.

\subsection{Effect of Rayleigh number}

The influence of Rayleigh number $R a$ (from $10^{3}$ to $10^{6}$ ) on streamlines and isotherms for the present configuration at $\operatorname{Pr}=0.7, \mathrm{Ha}=50$ and $\phi=0$ has been presented in Fig. 4. From the figure it is seen that there are two vortexes for $R a=10^{3}$ near the left and right inclined wall of the trapezoidal cavity. The left and right vortex increases with the increasing $R a$ which represents greater strength of the flow. As $R a$ number increases the difference of temperature between the heated and cold wall also increases which leads density variation in the air within the cavity. The air circulates strongly as a result the vortex inside the cavity increases.

Corresponding isotherms are shown in the Fig. 4 which represents the temperature field in the flow region. The patterns of isotherms are linear and look like parallel near the rectangular body for $R a=10^{3}$. These lines become more curved from the top wall of the left and right corner to the bottom wall because of growing $R a$. The isothermal lines become dense near the bottom of the heated body and top wall of the left and right corner for larger values of $R a$ because of higher temperature gradient which represent greater heat flow. As $R a$ number increases the difference of temperature between the heated and cold wall also increases. The air within the cavity transfer more heat and changes its heat flow pattern.

\subsection{Effect of Hartmann number}

Fig. 5(a) shows the effect of various $\mathrm{Ha}$ (from 0 to 70) on the flow field at $\mathrm{Ra}=10^{5}, \mathrm{Pr}$ $=0.7$, and $\phi=0$. In the absence of magnetic field the streamlines form a circulatory cell including the heated body at the left side and another cell at the right side of the heated body. As the Hartmann number increases the left and right cell losses their strength and decreases in size. Their shape also changes from blunt to sharp. It is known that magnetic field retards the fluid flow. So when $\mathrm{Ha}$ increases velocity decreases and the vortex inside cavity become smaller.

Fig. 5(b) shows the temperature field in the flow region. The isothermal lines become more curved from the top to bottom wall when $H a=0$. The isothermal lines become less dense and linear from top to bottom as Hartmann number increases. When Ha increases, heat flow changes its pattern because magnetic field retards the heat transfer. 

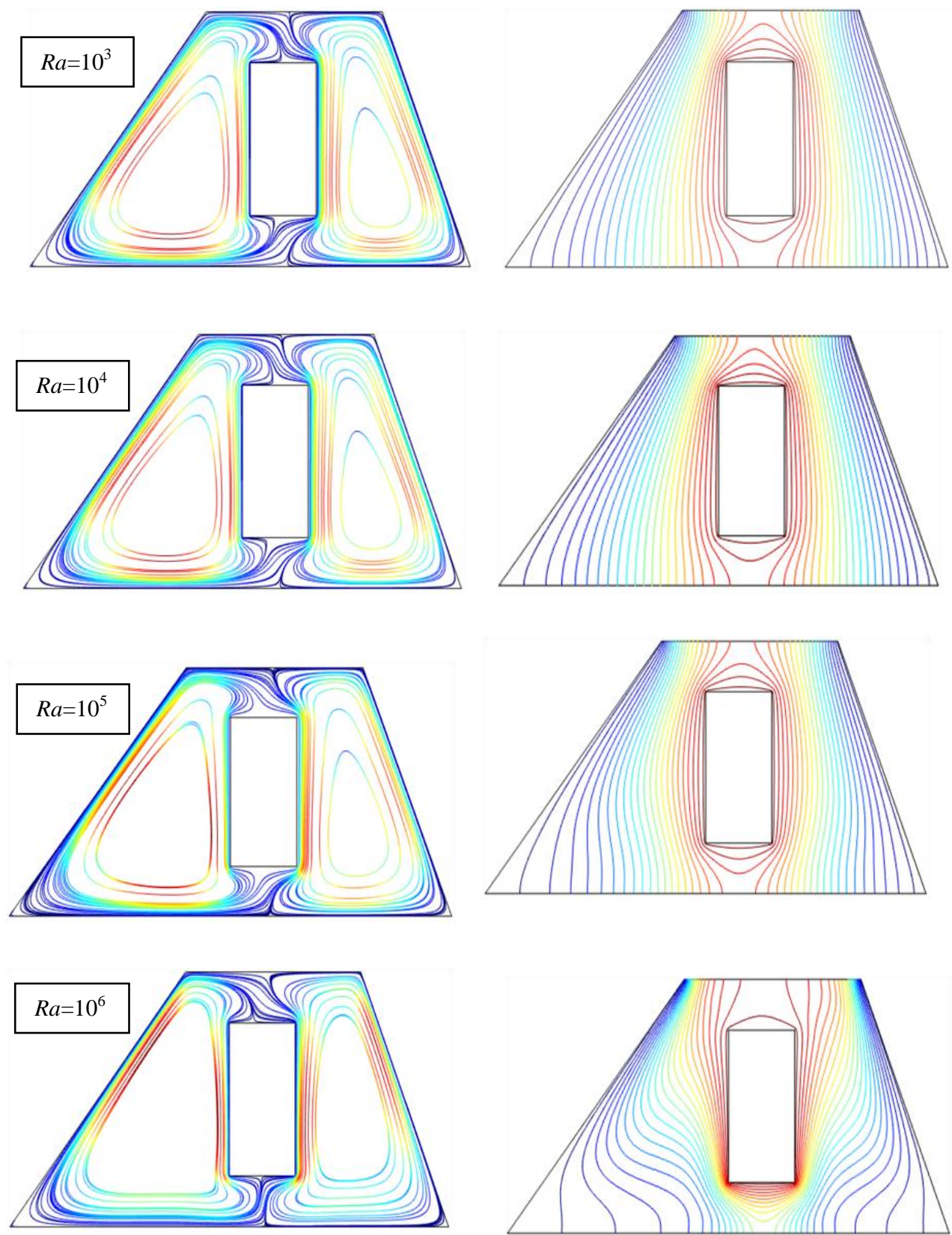

(a) Streamline

(b) Isotherm

Fig. 4. Effect of various Rayleigh number $R a$ on (a) streamlines and (b) isotherms [Hartmann number $\mathrm{Ha}=50$, Prandtl number $\operatorname{Pr}=0.7$, Inclination angle of the magnetic field $\phi=0^{\circ}$ ]. 

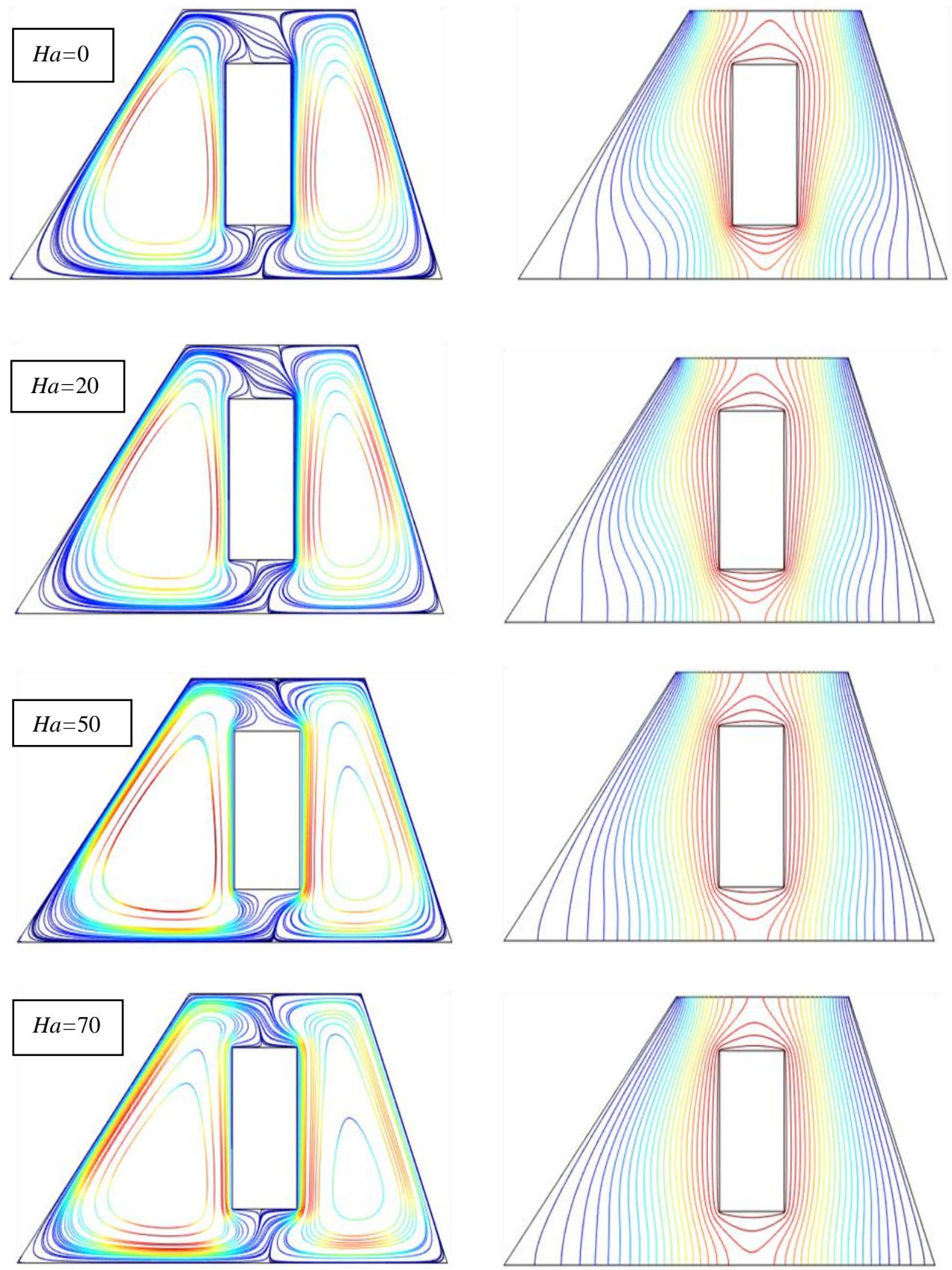

(a) Streamline

(b) Isotherm

Fig. 5. Effect of various Hartmann number $\mathrm{Ha}$ on (a) streamlines and (b) isotherms [Rayleigh number $R a=10^{5}$, Prandtl number $\operatorname{Pr}=0.7$, inclination angle of the magnetic field $\phi=0^{\circ}$ ]. 

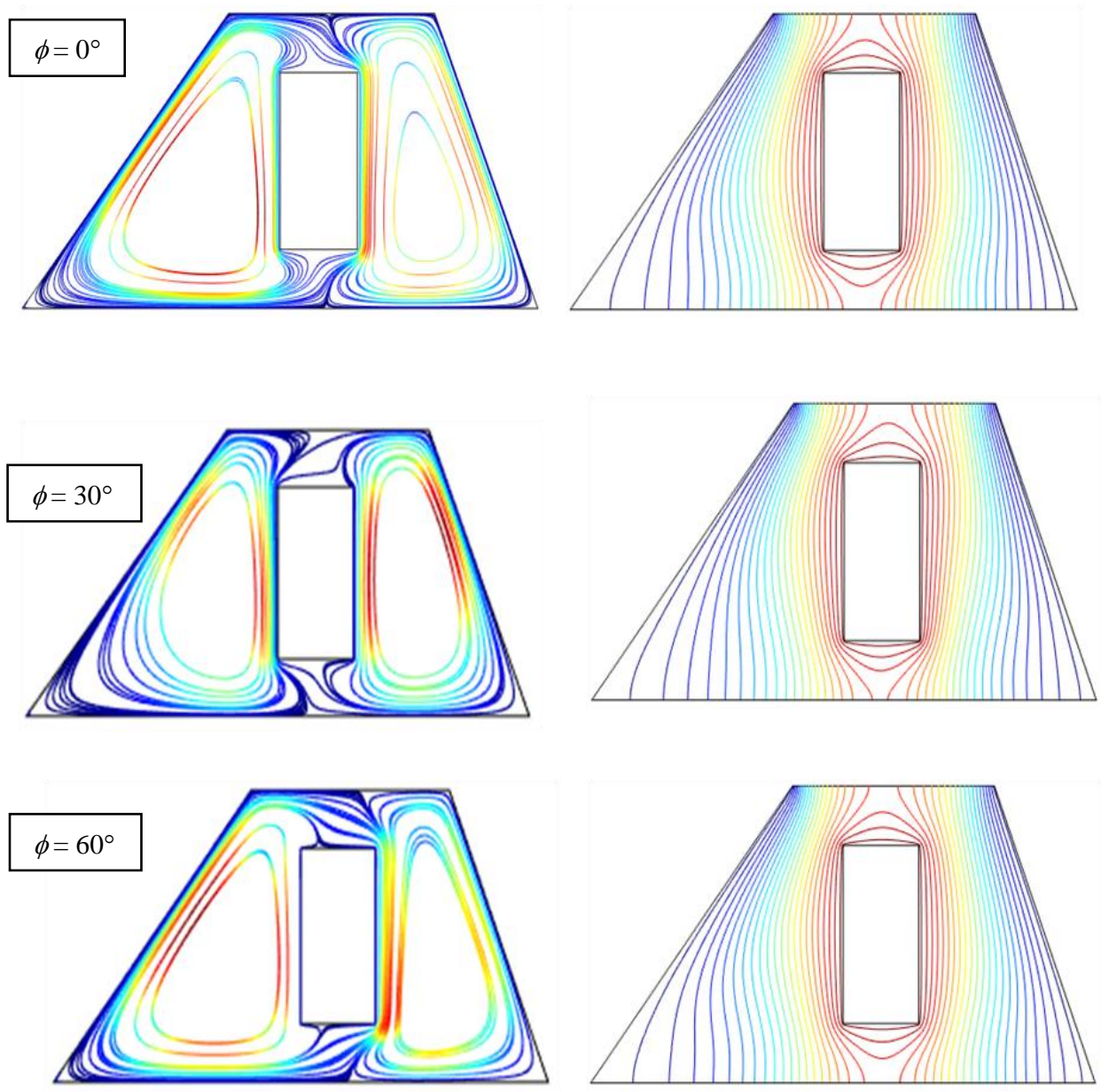

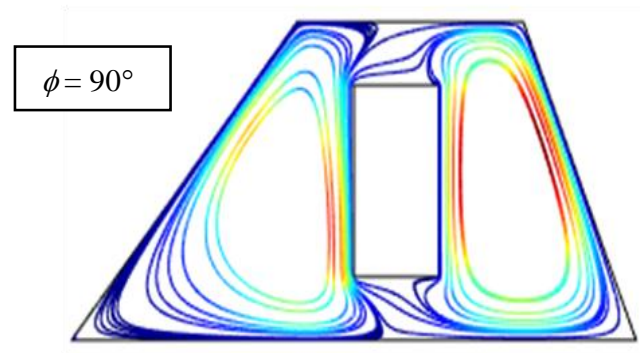

(a) Streamline

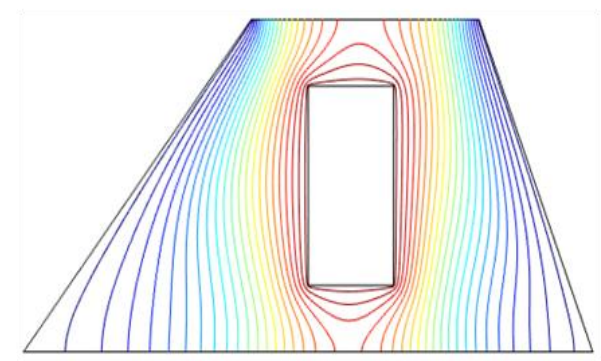

(b) Isotherm

Fig. 6. Effect of various Inclination angle of the magnetic field $\phi$ on (a) streamlines and (b) isotherms [Rayleigh number $R a=10^{5}$, Prandtl number $\operatorname{Pr}=0.7$, Hartmann number $\mathrm{Ha}=50$ ]. 


\subsection{Effect of orientation of magnetic field}

The effect of different orientation of magnetic field $\phi$ on the flow and thermal field at $R a$ $=10^{5}, \mathrm{Pr}=0.7$ and $\mathrm{Ha}=50$ are depicted in Fig. 6. It can be seen from Fig. 6(a) that the effect of the magnetic field direction is significant on the flow structure. When the direction of the external magnetic field $\left(\phi=0^{\circ}\right.$ and $\left.60^{\circ}\right)$ then the left cell include the heated body whereas at $\left(\phi=30^{\circ}\right.$ and $\left.90^{\circ}\right)$ the right cell includes the heated body. The higher flow strength is seen for $\phi=30^{\circ}$ and $90^{\circ}$ than that of $\phi=0^{\circ}$ and $60^{\circ}$. When the value of the angle of external oriented magnetic field is $0^{\circ}$ then the magnetic field has strongest effect. Changing the angle of external magnetic field from horizontal to vertical, the flow pattern is changed differently. The influence of the magnetic field on flow pattern is apparent from these figures.

The corresponding temperature field shows that the trends of isothermal lines are similar for all cases. The temperature field is affected with the orientation of the magnetic field like the flow field. Greater temperature gradient is observed at $\phi=30^{\circ}$ and $90^{\circ}$ which indicate higher heat transfer rate and lower heat transfer rate at $\phi=0^{\circ}$ and $60^{\circ}$.

The average Nusselt number is plotted as a function of different Rayleigh number, Hartmann number and orientation of magnetic field effect as shown in Fig.7 while keeping Prandtl number fixed at $P r=0.7$. It is observed that $\mathrm{Nu}$ rises with increasing $R a$ and decreases with increasing $\mathrm{Ha}$. The average Nusselt number changes differently with the rising of the angle of external magnetic field. The maximum and minimum heat transfer rate is obtained for the highest $\mathrm{Ra}$ and $\mathrm{Ha}$ respectively. The lowest $\mathrm{Nu}$ is found at $\phi=0^{\circ}$ and highest at $\phi=30^{\circ}$.
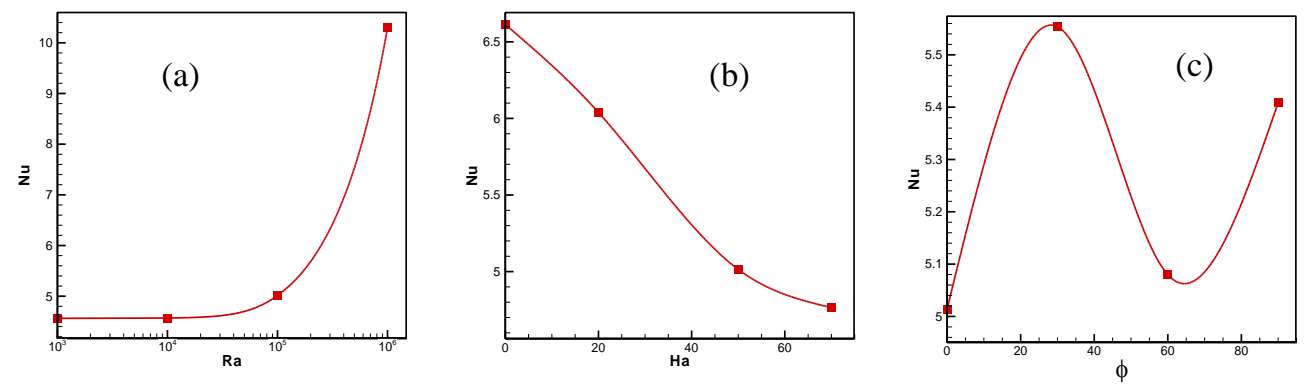

Fig. 7. The variation of average Nusselt number ( $N u$ with (a) different Rayleigh number $(\mathrm{Ra})$ at $\mathrm{Ha}$ $=50$ and $\phi=0^{\circ}$, (b) Different Hartmann number (Ha) at $R a=10^{5}$ and $\phi=0^{\circ}$ and (c) Different angle of orientation of magnetic field ( $\phi$ ) at $\mathrm{Ra}=10^{5}$ and $\mathrm{Ha}=50$.

\section{Conclusion}

The effects of magnetic field parameter $H a$, Rayleigh number $R a$ and orientation of magnetic field $\phi$ on flow and temperature field have been studied in detail in a trapezoidal cavity with a heated rectangular body inside. From the present investigation the following conclusions may be drawn: 
- The free convection parameter $R a$ has a significant effect on the flow and temperature fields. Increasing Rayleigh number increases the vortex in the streamline and average Nusselt number.

- Rising Hartmann number decreases both the vortex in the streamline and the heat flow.

- The increase of angle of the orientation of magnetic field has a great impact on both streamline and isotherms. Magnetic field has strongest effect on horizontal direction.

\begin{tabular}{|c|c|c|}
\hline \multicolumn{3}{|c|}{ Nomenclature } \\
\hline & specific heat $\left[\mathrm{Jkg}^{-1} \mathrm{~K}^{-1}\right]$ & $u, v \quad$ velocity components $\left[\mathrm{ms}^{-1}\right]$ \\
\hline & acceleration due to gravity $\left[\mathrm{ms}^{-2}\right]$ & $U, V$ dimensionless velocity components \\
\hline$k$ & thermal conductivity $\left[\mathrm{Wm}^{-1} \mathrm{~K}^{-1}\right]$ & $x, y \quad$ distance along $x$ - and $y$-coordinates \\
\hline$L$ & length of the base and height [m] & $X, Y$ dimensionless distance along $x$ - and \\
\hline $\mathrm{Nu}$ & Nusselt number & coordinates \\
\hline & dimensional pressure $[\mathrm{Pa}]$ & \\
\hline & dimensionless pressure & Greek symbols \\
\hline $\operatorname{Pr}$ & Prandtl number & $\alpha \quad$ thermal diffusivity $\left[\mathrm{ms}^{-2}\right]$ \\
\hline $\mathrm{Ra}$ & Rayleigh number & kinematic viscosity of the fluid $\left[\mathrm{m}^{2} \mathrm{~s}^{-1}\right]$ \\
\hline $\mathrm{Ha}$ & Hartmann number & dimensionless temperature \\
\hline & temperature $[\mathrm{K}]$ & density $\left[\mathrm{kgm}^{-3}\right]$ \\
\hline B & magnetic field & Stream function \\
\hline & cold temperature & Thermal expansion coefficient $\left(\mathrm{K}^{-1}\right)$ \\
\hline & heat temperature & \\
\hline
\end{tabular}

\section{Acknowledgments}

This research would not be possible without the support of Department of Mathematics, Bangladesh University of Engineering and Technology (BUET).

\section{Reference}

1. M. S. Hossain, M. A. Alim, and K. H. Kabir, Am. J. Appl. Math. Stat. 4(5), 161 (2016).

2. M. H. Esfe, A. A. A. Arani, W. M. Yan, H. Ehteram, A. Aghaie, and M. Afrand, Int. J. Heat Mass Transfer 92, 76 (2016).

3. S. Parvin and A. Akter, Procedia Eng. 194, 421 (2017). https://doi.org/10.1016/j.proeng.2017.08.166

4. A. Akter and S. Parvin, J. Eng. Math. Stat. 1(2,3), 1 (2017).

5. F. Selimefendigil, H. F. Öztop, and N. Abu-Hamdeh, Entropy 18(2), 43 (2016).

6. T. S. Lee, Numer. Heat Transfer A 19, 487 (1991). https://doi.org/10.1080/10407789108944861

7. M. Hasanuzzaman, H. F. Öztop, M. M. Rahman, N. A. Rahim, R. Saidur, and Y. Varol, Int. Commun. Heat Mass Transfer 39(9), 1384 (2012). https://doi.org/10.1016/j.icheatmasstransfer.2012.08.009

8. R. Nasrin and S. Parvin, Heat Mass Transfer 39, 270 (2012). https://doi.org/10.1016/j.icheatmasstransfer.2011.11.004

9. R. Ellahi, Appl. Math. Modell 37(3), 1451 (2013). https://doi.org/10.1016/j.apm.2012.04.004 
10. M. Sheikholeslami, M. Gorji-Bandpy, and D. D. Ganji, Energy 60, 501 (2013). https://doi.org/10.1016/j.energy.2013.07.070

11. S. Ostrach, Adv. Heat Transfer 8, 161 (1972). https://doi.org/10.1016/S0065-2717(08)70039-X

12. S. Parvin and R. Nasrin, J. Sci. Res. 4(2), 337 (2012). https://doi.org/10.3329/jsr.v4i2.8142

13. S. Parvin, R. Nasrin, M. A. Alim, and N. F. Hossain, J. Sci. Res. 5(1), 67 (2013).

14. M. M. Molla and M. M. A. Sarker, Natural Convection Flow in a Square Cavity with Temperature Dependent Heat Generation - Proceedings of $3^{\text {rd }}$ BSME-ASME Int. Conf. on Thermal Eng., Bangladesh.

15. O. Aydin and A. U. Nal, Int. J. Heat Mass Transfer 42, 2345 (1999). https://doi.org/10.1016/S0017-9310(98)00319-6

16. Z. Y. Zhong, K. T. Yang, and J. R. Lloyd, J. Heat Transfer 107, 133 (1985). https://doi.org/10.1115/1.3247369

17. R. Chowdhury, S. Parvin, and M. A. H. Khan, Heat Generation Effect on Natural Convection Flow in a Rhombic Shape Cavity Containing a Rectangular Block - Proceedings of the Int. Conf. on Mechanical Eng. and Renewable Energy 2015 (ICMERE2015), Bangladesh. ICMERE2015-PI-029, (2015).

18. M. A. Sheremet, H. F. Oztop, I. Pop, and N. Abu-Hamdeh, Entropy 18, 9, (2015).

19. A. El Jery , N. Hidouri, M. Magherbi and A. B. Brahim, Entropy, 12, 1391 (2010). https://doi.org/10.3390/e12061391

20. T. Hayat, A. Shafiq, A. Alsaedi, and S. Asghar, AIP Adv. 5, 087108 (2015). https://doi.org/10.1063/1.4928321

21. N. Sandeep and V. Sugunamma, World Appl. Sci. J. 22(7), 975 (2013).

22. E. Baskaya, G. Komurgoz, and I. Ozkol, Entropy, 19, 377 (2017). https://doi.org/10.3390/e19070377

23. Z. Mehrez, A. El Cafsi, A. Belghith, and P. Le Quéré, J. Magn. Magn. Mater. 374, 214 (2015). https://doi.org/10.1016/j.jmmm.2014.08.010

24. S. Parvin and R. Nasrin, Nonlinear Analysis: Modelling and Control 16, 89 (2011). 